\title{
Relativistic classical Monte Carlo simulations of stabilization of hydrogenlike ions in intense laser pulses
}

\author{
L. N. Gaier* and C. H. Keitel ${ }^{\dagger}$ \\ Theoretische Quantendynamik, Fakultät für Physik, Albert-Ludwigs-Universitat Freiburg, Hermann-Herder-Strasse 3, \\ D-79104 Freiburg, Germany
}

(Received 16 July 2001; revised manuscript received 5 September 2001; published 10 January 2002)

\begin{abstract}
The relativistic classical dynamics of hydrogenlike ions is investigated numerically in the presence of intense high-frequency laser pulses of intensity well above $10^{16} \mathrm{~W} \mathrm{~cm}^{-2}$ and various pulse lengths up to several hundred cycles. With rising effective charge $Z$, the stabilized electron dynamics is shown to be increasingly governed by the joint interplay of Coulomb attraction and Lorentz force in the laser-propagation direction, involving also so-called magnetic recollisions. With regard to the ionization behavior, the varying stabilization with increasing $Z$ can be largely described by scaling the angular frequency and electric field strength by $Z^{2}$ and $Z^{3}$, respectively, however, clear signatures appear also due to the increased role of relativity. For long pulses and slowly entering the relativistic regime, the enhanced adiabaticity of the pulse turn-on has insufficient influence because the breakdown of stabilization due to the Lorentz force will still be stronger due to the dominating role of the necessarily increased interaction time with the superintense laser field.
\end{abstract}

DOI: $10.1103 /$ PhysRevA.65.023406

PACS number(s): $32.80 . \mathrm{Rm}, 42.50 . \mathrm{Hz}, 42.65 . \mathrm{Ky}$

\section{INTRODUCTION}

Ever since its discovery by Gavrila and Kamiński [1], and its first demonstration in simple quantum models by $\mathrm{Su}$ and co-workers [2], the stabilization phenomenon has been the subject of wide research in intense laser-atom interaction. In this context, stabilization refers to an increase in laser intensity giving rise to a decrease in the probability of photoionization of an atomic system. Theoretically it has been investigated both classically and quantum mechanically, mainly in one [2-9], but also in two [10-13] and three [14-17] dimensions. The effect has been linked mainly to smoothly turned-on intense laser pulses of high frequency $\omega$ exceeding the Kepler frequency $\nu_{k}$ of the atomic system, with ongoing discussion, however, on the precisely required frequency regime $[3,6,11,15-21]$. Experimental evidence for stabilization also exists [22], in spite of the lack of efficient highfrequency intense laser sources up to date.

The benchmark atomic system - the hydrogen atom-has almost exclusively been the subject of all this research. More complex atomic and molecular systems have been considered to a far smaller extent due to the significant complications inherent in multielectronic systems (see here, e.g., [23$25]$ and for a review involving correlation and stabilization effects [26]). A further means of gaining a generalized picture of stabilization is via studying the behavior of isoelectronic ions (such as $\mathrm{He}^{+}, \mathrm{B}^{4+}$, and $\mathrm{Ne}^{9+}$ as considered here), which are relatively simple to model on the atomic side. They, however, involve a complex relativistic dynamics if laser pulses are applied with a field strength comparable to that via the ionic potential. As yet, less work has been carried out here and has been mostly restricted to the "low-

\footnotetext{
*On leave from The Blackett Laboratory, Imperial College, London SW7 2BZ, UK. Email address: lucien.gaier@ic.ac.uk

${ }^{\dagger}$ Email address: keitel@uni.freiburg.de
}

frequency" (near-optical) regime (see [27-30] and references therein).

Stabilization is widely believed to break down in the relativistic regime, especially due to the Lorentz force $[15,17,10,11]$, which is not confirmed with one-dimensional models [7,9] and by [20]. In the cases with breakdown of stabilization with large Lorentz force, the pulse length was kept constant with increasing laser intensity, which means that the adiabaticity is reduced at the same time. All relativistic studies of stabilization $[7-10,15,17]$ and those beyond the dipole approximation [11,12] employ rather short laser pulses with respect to the achieved maximal intensity, and are often in lower dimensions, which is simply due to the numerical difficulty inherent in this regime. It is, however, also important to study relativistic electron dynamics and stabilization and ionization without these constraints because in the nonrelativistic regime, adiabaticity, and therefore pulses with long turn-on stages, play an important role. At present this appears impossible with a fully quantummechanical relativistic code. Classical mechanics is computationally easier to employ, allowing for 3D relativistic dynamics, longer pulses, the inclusion of the Lorentz-force term, and practically any value for laser frequency and intensity. Although the classical picture often turned out to be a poor way of treating an atomic system, with such high intensities being used, the classical picture has been shown to be a reasonable approximation for ionization and stabilization dynamics [16,31]. While intrinsic quantum effects such as quantum interferences and spin signatures are, in general, of smaller significance in this case, the uncertainty of an electron wave packet, however, does play a large role but may well be modeled classically via Monte Carlo simulations $[32,33,15,17]$.

In this article we study the interaction of multiply charged isoelectronic ions with very intense high-frequency laser pulses with emphasis on the onset of stabilization and relativistic signatures. The treatment is classical, fully relativistic, i.e., also without dipole approximation, and in all three 

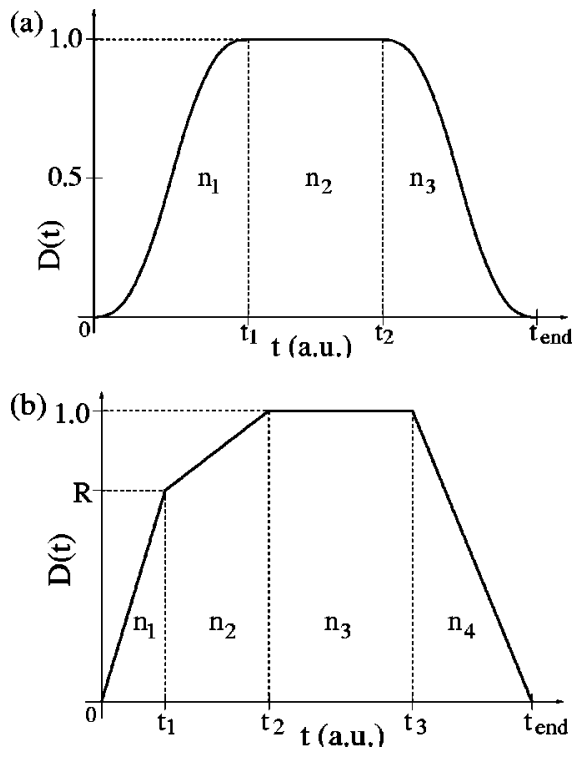

$D(t)= \begin{cases}\sin ^{2}\left(\frac{\omega t}{4 n_{1}}\right) & , 0 \leq t \leq t_{1} \\ 1.0 & , t_{1}<t<t_{2} \\ \sin ^{2}\left(\frac{\omega\left(t-t_{2}\right)}{4 n_{3}}+\frac{\pi}{2}\right) & , t_{2} \leq t \leq t_{\text {end }} \\ 0.0 & , \text { otherwise }\end{cases}$

$D(t)= \begin{cases}\frac{R t}{t_{1}} & , 0 \leq t \leq t_{1} \\ \frac{(1-R) t+\left(R t_{2}\right)-t_{2}}{t_{2}-t_{3}} & , t_{1}<t \leq t_{2} \\ 1.0 & , t_{2}<t<t_{3} \\ \frac{\left(t-t_{3}\right)}{t_{3}-t_{\text {end }}}+1 & , t_{3} \leq t<t_{\text {end }} \\ 0.0 & , \text { otherwise }\end{cases}$
FIG. 1. The pulse shapes used throughout this paper are described here. (a) shows the extensively used sine-squared envelope function, with $D(t)$ as a function of laser-atom interaction time $t$. $D(t)$ takes only values between 0 and 1. (b) shows the less wellknown two-stage envelope function, similarly suggested by $\mathrm{Ku}-$ lander, Schafer, and Krause [14]. $R$ is the $D(t)$ value at which the second stage of the field ramping begins. dimensions. We consider charge states of the ions between $Z=1$ and $Z=10$ and study the role of adiabaticity with turn-on lengths ranging from 1 to 300 cycles, with $\sin ^{2}$ and linear ramping. Also investigated are "two-stage" turn-on phases, where the field ramping is broken into two distinct sections before and after entering the relativistic regime with different ramping rates. The motivation here is to evolve the system through the regime of lower laser intensities and higher ionization ("death-valley regime" [19]) rather quickly while to move it slowly into the relativistic regime. We display stabilized electron trajectories and place emphasis on the reduced dimensions of the electron motion for higher charged ions and particularly on recollisions in the direction of propagation of the laser pulse. The ionization and stabilization behavior is similar in ions if the applied laser frequency and electric-field strength are scaled by $Z^{2}$ and $Z^{3}$, respectively, apart from a notable reduction of stabilization due to relativity. The enhanced adiabaticity for pulses with significantly longer turn-on phases into the relativistic regime are shown not to inhibit the breakdown of stabilization because the effect of the increased interaction time seems to be dominating.

The paper is structured as follows. We begin in Sec. II by discussing the classical model used to describe the laseratom or -ion interaction, as well as outlining the procedure behind the Monte Carlo averaging process and the determination of initial conditions. We then show and discuss the results concerning classical electron trajectories in Sec. III and ionization probabilities in Sec. IV of the various ions for different pulse parameters. We end with conclusions that can be drawn from our results.

\section{CLASSICAL MONTE CARLO SIMULATION}

We proceed by discussing the modeling of our classical atomic system and the efforts employed to mimic a true quantum system. Throughout this paper atomic units (a.u.) are used, and as such, high frequency, high intensity, and high electric field can all be thought of as being of the order of 1 a.u. or greater.

\section{A. The equation of motion}

The relativistic equation of motion for an electron in the Coulombic potential of the ion interacting with a laser pulse is as follows:

$$
\begin{aligned}
\frac{d}{d t} \frac{\dot{\mathbf{r}}}{\sqrt{1-(\dot{\mathbf{r}} / c)^{2}}}= & \mathbf{F}=\mathbf{E}_{0} D(t) \cos (\eta)+\frac{\dot{\mathbf{r}}}{c} \times \mathbf{H}_{0} D(t) \cos (\eta) \\
& -\frac{Z \mathbf{r}}{\left|\mathbf{r}^{3}\right|}
\end{aligned}
$$

$\mathbf{F}$ is the force acting on the electron. $\mathbf{r}$ is defined as electron position in Cartesian space with respect to the comparatively far more massive nucleus, fixed at the origin, with $\gamma=[1$ $\left.-(\dot{\mathbf{r}} / \mathbf{c})^{2}\right]^{-1 / 2}$ being the familiar relativistic gamma factor and the dot on vectors referring to a derivative in time. $\mathbf{E}_{0}$ and $\mathbf{H}_{0}$ are the electric-field and magnetic-field components of the laser field, in the $x$ and $y$ directions respectively, with maximal field strength $E_{0}=\left|\mathbf{E}_{0}\right|=\left|\mathbf{H}_{0}\right|$ in atomic units, $c$ being the speed of light in a vacuum. The laser phase is defined as $\eta=\omega t-k z$, where $\omega$ is the laser frequency, $t$ is the time, $k=\omega / c$ is the laser wave number, and $z$ is the propagation direction. The laser is linearly polarized in the $x$ direction throughout the paper.

The pulse shapes [described by the envelope function $D(t)]$ used are either of the two forms: "sine squared" or "linear two stage" (see Fig. 1). The sine-squared pulse shape, written as $\sin ^{2}\left(n_{1}-n_{2}-n_{3}\right)$, describes a pulse with sine-squared turn-on and turn-off stages of duration $n_{1}$ and $n_{3}$ cycles, respectively, and $n_{2}$ cycles at constant maximum value equal to unity. The two-stage envelope functions have linear turn-ons and turn-offs, with the turn-on being broken into two stages of length $n_{1}$ and $n_{2}$ cycles, constant maximum value 1 for $n_{3}$ cycles, and turn-off of duration $n_{4}$ cycles.

Rearranging Eq. (1) to give $\ddot{\mathbf{r}}$ only on the left-hand side results in $\ddot{\mathbf{r}}=\left[\mathbf{F}-\dot{\mathbf{r}}(\dot{\mathbf{r}} \cdot \mathbf{F}) / c^{2}\right] / \gamma$. By inserting $\mathbf{F}$ and evaluating components, an electron trajectory can be plotted for a 


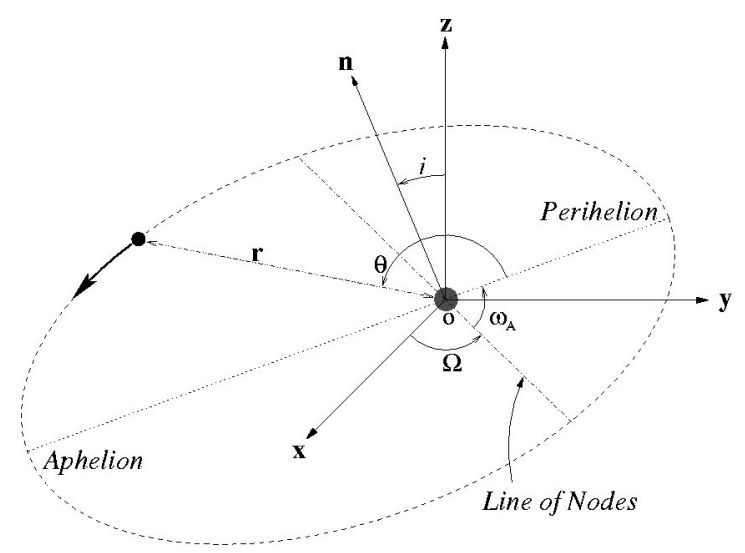

FIG. 2. A Kepler orbit. The line of nodes is the line of intersection between the orbital plane and the $x-y$ plane. Characteristic parameters are $i$ (inclination of orbit), $\Omega$ (longitude of ascending node), $\alpha$ (mean anomaly), $c$ (eccentricity of orbit), $\omega_{A}$ (argument of perihelion), and $\mathbf{n}$ (normal to orbital plane).

given set of initial conditions. There are various variations on these equations, which are also considered here. First is the case of a regularized soft-core potential [34] $V_{\mathrm{SC}}$ $=Q Z / \sqrt{r^{2}+s^{2}}$, where $s$ is the smoothing parameter and $Q$ is a constant that ensures the electron has the correct initial energy. Second, is the case where the envelope function $D$ varies with $\eta$ rather than just $t$. This allows the whole pulse shape to move along the propagation axis. However, it tends to be computationally more time consuming to model because of the additional spatial dependence in the argument of the envelope function. This restricts us in the calculations to fewer trajectories in the same time, i.e., a smaller accuracy of the result. $D(t)$ is shown later to be a good approximation to $D(\eta)$ for the employed range of parameters.

\section{B. The initial Monte Carlo ensemble}

Qualitatively, an atomic or ionic system is best represented quantum mechanically. However, we may mimic the uncertainty of a quantum-mechanical wave packet in a classical model of an atom through the assumption that the atom be represented by an ensemble of electrons in a microcanonical distribution. This is a well-known and experimentally confirmed procedure (see, e.g., [32]), which will merely be presented here for completeness for the generalized case of hydrogenic ions. The initial energy distribution function $\rho\left(\mathcal{E}_{0}\right)$ of the electron is defined as usual as $\rho\left(\mathcal{E}_{0}\right) \propto \delta\left(\mathcal{E}_{0}\right.$ $-\mathcal{U})[32,33]$, where $U=-Z^{2} / 2$ is the ionization energy of the single electron in isoelectronic systems $\left(\mathrm{H}, \mathrm{He}^{+}\right.$, etc.).

The only constraint placed upon the unperturbed electronic orbit is that the energy remain constant. All other electron properties, such as position and momentum, can vary accordingly from system to system. With the addition of the fact that the force acting on the electron due to the nucleus is central in nature, the result is the electron following a bound Kepler orbit (see Fig. 2). Such an orbit is described using the Kepler equation $\alpha=\psi-e \sin (\psi), \alpha, e$, and $\psi$ are the mean anomoly, eccentricity, and eccentric anomaly of the orbit, respectively. They further relate to the true anomaly
( $\theta)$ of the orbit through the following: $\tan (\theta / 2)$ $=\sqrt{(1+e) /(1-e)} \tan (\psi / 2)$. Kepler's equation is not solvable analytically, although it is possible to solve using iterative methods (among others). In this case, we found $\psi$ for any $\alpha$ and $e$ using a Newton-Raphson iteration method. For a Kepler orbit to be described uniquely requires six initial conditions: the internal energy of the atom $U$, and five random values for the following Kepler orbit elements (see Fig. 2 ). These are chosen with equal probability within the given ranges

$$
\begin{gathered}
0 \leqslant \alpha \leqslant 2 \pi, \quad 0 \leqslant e^{2} \leqslant 1, \quad-1 \leqslant \cos i \leqslant 1, \\
0 \leqslant \Omega \leqslant 2 \pi, \quad 0 \leqslant \omega_{A} \leqslant 2 \pi .
\end{gathered}
$$

Having determined all these variables and conditions, it is now possible to obtain the electron's initial properties with the following via [33]

$$
\begin{gathered}
l^{2}=\frac{Z^{2} M\left(e^{2}-1\right)}{2 U}, \\
a=\frac{Z(e \cos \psi-1)}{2 U}, \\
p^{2}=2 M\left[\frac{Z}{a}+\frac{Z^{2}\left(1-e^{2}\right)}{4 U a^{2}}+U\right],
\end{gathered}
$$

where $l, a$, and $p$ are angular momentum, radial distance, and linear momentum, respectively. Finally, these properties are used to find the initial conditions of the electron,

$$
\begin{gathered}
r_{x}(0)=a\left[\cos \Omega \cos \left(\omega_{A}+\theta\right)-\sin \Omega \cos i \sin \left(\omega_{A}+\theta\right)\right], \\
r_{y}(0)=a\left[\sin \Omega \cos \left(\omega_{A}+\theta\right)+\cos \Omega \cos i \sin \left(\omega_{A}+\theta\right)\right], \\
r_{z}(0)=a\left[\sin i \sin \left(\omega_{A}+\theta\right)\right], \\
\dot{r}_{x}(0)=\frac{1}{a}\left\{p r_{x}(0)-l\left[\cos \Omega \sin \left(\omega_{A}+\theta\right)\right.\right. \\
\left.\left.+\sin \Omega \cos i \cos \left(\omega_{A}+\theta\right)\right]\right\} \\
\dot{r}_{y}(0)=\frac{1}{a}\left\{p r_{y}(0)-l\left[\sin \Omega \sin \left(\omega_{A}+\theta\right)\right.\right. \\
\left.\left.+\cos \Omega \cos i \cos \left(\omega_{A}+\theta\right)\right]\right\} \\
\dot{r}_{z}(0)=\frac{1}{a}\left\{p r_{z}(\theta)+l\left[\sin i \cos \left(\omega_{A}+\theta\right)\right]\right\} .
\end{gathered}
$$

Note that the initial conditions are obtained nonrelativistically. This is allowable for hydrogen and the lighter ions $(Z \leqslant 10)$ as the nuclear core does not accelerate the electron to relativistic speeds. Heavier ions, however, require relativistic treatments, and the effect of the nonrelativistic treatment can be seen at low electric-field strengths where ionization probability never reaches zero [for example, see Fig. 4(d)]. For this reason, ions heavier than neon $(9+)$ are not considered in this paper. 

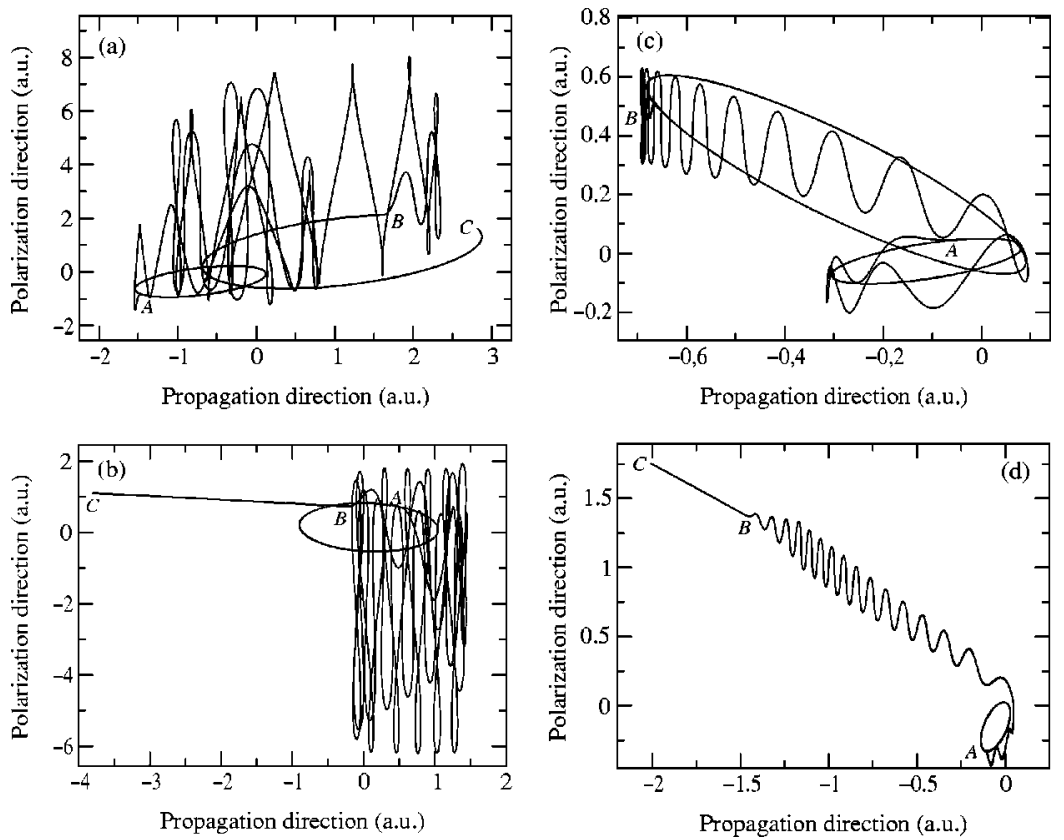

FIG. 3. Typical trajectories of initially bound electrons before (up to point $A$ ), during ( $A$ $\rightarrow B)$, and after $(B \rightarrow C)$ the laser pulse has interacted with the atomic systems. (a) and (b) are for a hydrogen atom, while (c) and (d) are for boron $(4+)$. (a) and (c) show trajectories where the electrons, initially in bound Kepler orbits, remain bound to the nuclear cores at the end of the pulse $(B)$, albeit in an excited Kepler orbit. (b) and (d) show the electrons, which ionized during the pulse, traveling away from the nucleus. The pulses used had two-stage 1-10-4-4 envelopes, with $E_{0}=100.0$ a.u., $\omega=5.0$ a.u. for the $H$ case, and $E_{0}=2500.0$ a.u., $\omega=125.0$ a.u. for $\mathrm{B}^{4+}$.

\section{The ionization probabilities}

The initially prepared atomic or ionic systems are subjected to a laser pulse, in which the electron's classical trajectory can be plotted. In order to determine its state at the end of the pulse (i.e., still bound or ionized), we evaluated the electron's energy $\mathcal{E}$ using the following expression:

$$
\mathcal{E}=-\frac{Q Z}{\sqrt{\mathbf{r}^{2}+s^{2}}}+c^{2}\left[\frac{1}{\sqrt{1-(\dot{\mathbf{r}} / c)^{2}}}-1\right]
$$

with the electron being found still bound if $\mathcal{E}<0$ and ionized if $\mathcal{E}>0$. The Monte Carlo simulation is completed by considering many such trajectories, from which we obtain a probability of ionization occurring through the interaction of the laser pulse with the isoelectronic system. Throughout the paper, averaging took place over 1000 such classical trajectories. This procedure is not in itself new-it has been implemented many times (see, for example, [17,32,35-37]). However, to the authors' knowledge, the method has only ever been used in the case of hydrogen. Equations (2)-(4) are for the more general case of hydrogenlike ions of nuclear charge $Z$.

The classical Monte Carlo simulation is inherently an averaging technique, and as such there is always a certain "error" in the probability values obtained by the method. No two random selections of initial conditions will give identical probability values for a given set of system parameters. This leads to jagged features in the curves, something that can be minimized with increasing the number of trajectories. By looking at a number of results for the same system parameters, we found that ionization probability values for any particular graph can vary by $0.5-0.6 \%$ at the extreme values of $E_{0}$ tested, and by $0.9-1.1 \%$ within the stabilization feature (in the case of averaging over 1000 trajectories).

\section{TRAJECTORIES OF IONIZED AND STILL-BOUND ELECTRONS}

A look at the classical electron's trajectory during the interaction can help establish an intuitive picture of the underlying physics. Figure 3 shows four such trajectories for various scenarios. The laser frequency in the boron $(4+)$ case was scaled by $Z^{2}$ from the hydrogen case in order to regain conditions necessary for stabilization. In all the graphs, it is possible to see the ground-state Kepler orbit followed by each electron before the interaction with the laser pulse. During the pulse interaction the electron follows a complex trajectory, dominated by the laser pulse. It is also possible to see in parts the electron moving back towards the core, in the negative laser propagation direction. Such "magnetic recollisions" [38] are due to the variation in the relative influence between laser Lorentz force and the attractive Coulomb force.

The difference in trajectories occurs after the pulse has passed. Still-bound electrons continue to orbit the nucleus in an excited state, while ionized electrons travel away from the nucleus in almost a straight line. The region of coordinate space visited by the electron during the interaction, in general, decreases with increasing nuclear charge. This comes about for various reasons. First, the ground-state orbit lies nearer the nucleus for heavier ions, so the electron starts closer to the core. Second, the attractive force due to the nucleus increases proportionately with $Z$, so the electron has greater difficulty escaping the nucleus. In Fig. 3, the electricfield strength was scaled with $Z^{2}$ (to reenter the stabilization regime), indicating that a scaling with a higher exponent is necessary to compensate for the enhanced attraction of the nucleus almost fully ( $Z^{3}$ as to be seen later). Lastly, the interaction time between the two cases is significantly shorter, simply due to the $Z^{2}$ scaling of the laser frequency (pulse length has remained constant).

A significant aspect of all the classical trajectories is the 

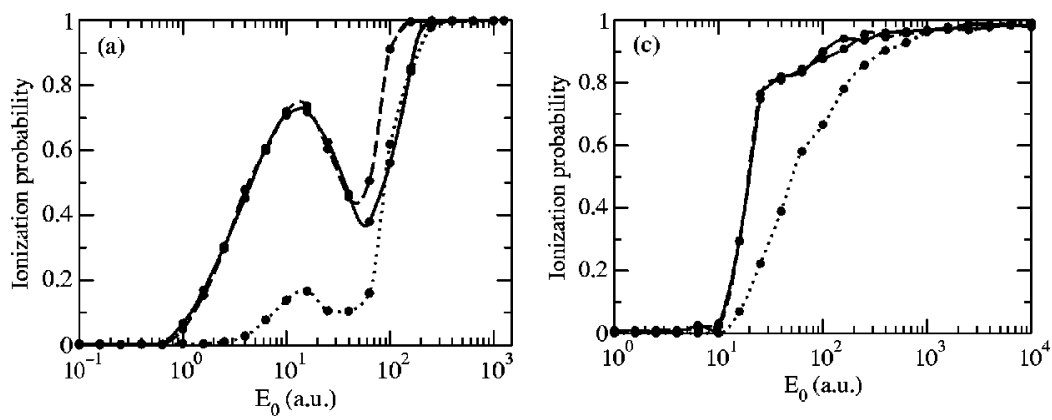

FIG. 4. Ionization probability against the maximum electric-field strength of the laser $\left(E_{0}\right)$ for the cases of (a) hydrogen, (b) helium $(+),(\mathrm{c})$ boron $(4+)$, and $(d)$ neon $(9+)$. Each graph depicts the cases where the nuclear core considered was either purely Coulombic (solid line), or soft core with $s^{2}=0.25$ (dotted line). The dashed line also represents the case of a Coulomb potential with the laser pulse now completely dependent on the phase $\eta$. The laser pulses used were $\sin ^{2}$ (3-4-3) type with frequency $\omega=5.0$ a.u. for all cases. Probabilities at each data point (shown by the dots) were obtained through averaging over 1000 Monte Carlo trajectories. The curve connecting each dot is a smoothed representation of the possible ionization-probability curve.
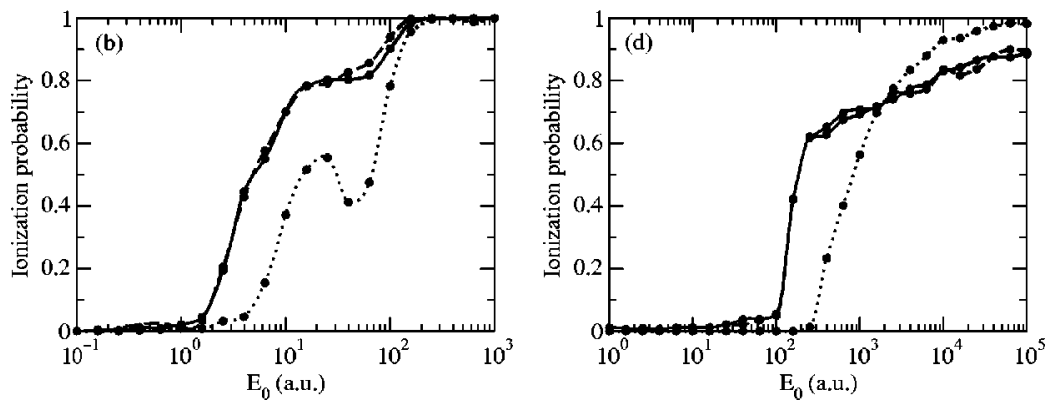

position of the electron at the end of the pulse. Often, an electron can end up driven many atomic units away from the nucleus and still remain bound (therefore lying in a Rydberg state), whereas it is also possible for the electron to lie very close to the nucleus, but with positive energy. This is an important distinction from quantum-mechanical calculations, where ionization is sometimes judged to occur when the electron has been absorbed by the grid boundaries of the system. The electron acts almost as a free particle far from the nucleus, which in turn means the electron gains little net energy during the laser-atom interaction. If the electron has negative energy when it enters this "almost-free" region, it is unlikely to gain enough energy to ionize until it recollides or rescatters with the nucleus.

\section{IONIZATION PROBABILITIES OF HYDROGEN AND ISOELECTRONIC IONS}

Having looked at individual trajectories, the next step is to consider the probability of ionization occurring during a particular interaction. Figure 4 illustrates the variation in ionization probabilities between hydrogen and hydrogenlike ions, each at the same laser frequency. Both Coulombic and softcore treatments are examined to illustrate the differences that occur for each model. Also shown is the case for a laser pulse where the envelope function is of the form $D(\eta)$ rather than $D(t)$. All show the same general trends, however, the stabilization feature decreases with increasing $Z$ and the curves shift towards higher field strengths. The removal of the stabilization features in the boron $(4+)$ and neon $(9+)$ cases are a result of the frequency of the laser light (in this case $\omega=5)$. Stabilization generally occurs when $\omega>\nu_{k}$, and this inequality is only satisfied for hydrogen $\left(\nu_{k}=1\right)$ and helium $(+)\left(\nu_{k}=4\right)$. For the heavier ions there is a plateauing in ionization probability for increasing field strengths. This can be explained by the electron being removed from the vicinity of the nucleus so suddenly, that there is little time for the electron to gain sufficient net energy to ionize. The shift of the main features to higher laser intensities comes naturally from the increased attractive force due to the nucleus.

The soft-core model, in general, lowers the ionization probabilities in the curves, and frequently acts to enhance stabilization features. This has been shown to occur for helium $(+)$, where the stabilization dip is much stronger than in the purely Coulombic model for $\omega=5.0$ a.u. This is more in line with quantum-mechanical calculations done thus far. It has been shown [16] that classical Coulomb models tend to overestimate ionization probabilities and usually require higher frequencies for stabilization to occur. Some argue that the principal quantum effect is the regularization of the nuclear potential. Further, a smoothed potential can be said to be an effective means of modeling the uncertainty in nuclear position, which results from giving the nucleus zero momentum [16]. The soft-core potential though was mainly introduced as a numerical tool to overcome the problem of modeling a singular potential, particularly for lowerdimensional models. In our case, however, it is not necessary, due to the chances of the electron hitting the nucleus in three-dimensional space being so small. We have also found that the hydrogen nucleus does not require smoothing at $\omega$ $=1.0$ a.u. for it to exhibit stabilization (which quantummechanical calculations predict at this frequency). This runs contrary to previous classical results [15-17], although the stabilization feature itself is rather small [see hydrogen case in Fig. 5(a)].

We note also from Fig. 4 that the differences in using a moving pulse envelope $D(\eta)$ rather than $D(t)$ are quite small for our parameters, particularly in comparison to the effect of the smoothing of the nuclear potential. The largest differences occur within the stabilization feature and for lower ionic charges. Due to the momentum transfer of the laser pulse to the electron in the laser-propagation direction, the electron is more likely to move in this direction rather 

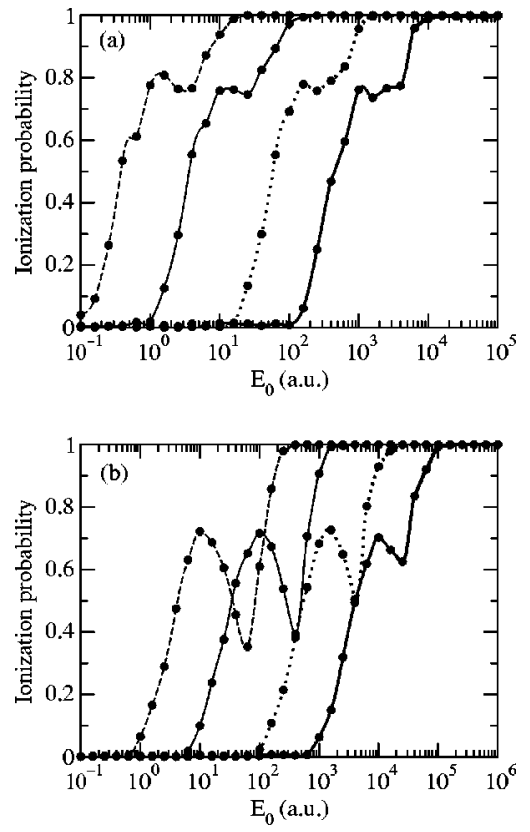

FIG. 5. Ionization probability as a function of electric-field strength for Coulombic hydrogen (dashed line), helium $(+)$ (thin solid line), boron $(4+)$ (dotted line), and neon $(9+)$ (thick solid line). Laser parameters are those as given Fig. 4 (with envelope function in terms of $l$ only), except for the laser frequency. In both graphs, the frequency has been scaled proportional to $Z^{2}$ in an attempt to reproduce the conditions necessary for stabilization to occur. In graph (a) $\omega=Z^{2}$ and in (b) $\omega=5 Z^{2}$.

than opposite to it. As a result, in the description via $D(\eta)$, most members of the electron ensemble move with the pulse and thus experience a longer turn-on phase than in the description via $D(t)$. There will also be a redshift of the laser frequency experienced by the electron in its own rest frame but this is also included in a description via $D(t)$. Both effects mean a reduction of stabilization; the effect of the enhanced interaction time in the "death valley" due to the effectively extended pulse length in the $D(\eta)$ case is visible in Fig. 4(a) by comparing the solid and dashed lines. This influence becomes smaller for higher charges of the ions because bound dynamics then takes place in the nearer vicinity of the ionic core.

The close relationship between hydrogen and the isoelectronic ions is clearly demonstrated in Figs. 5(a) and 5(b), which show a series of very similar curves, shifted towards higher $E_{0}$ values for the cases when the laser frequency is scaled proportional to $Z^{2}$. This scaling is employed to account for the increase in the Kepler frequency of the groundstate orbit. For the case of $\omega=Z^{2}$, all cases give the same amount of stabilization, with the electric-field strengths at which the stabilization feature occurs scaling approximately with $Z^{3}$ between hydrogen and the ions. The shifting to higher intensities is again due to the increased attractive force that the heavier ions exhibit. At these frequencies, larger laser forces are needed to disrupt the electronic orbit sufficiently for excitation and ionization to take place. However, in the case of $\omega=5 Z^{2}$, the curves differ more greatly. The stabilization features again begin at approximately the
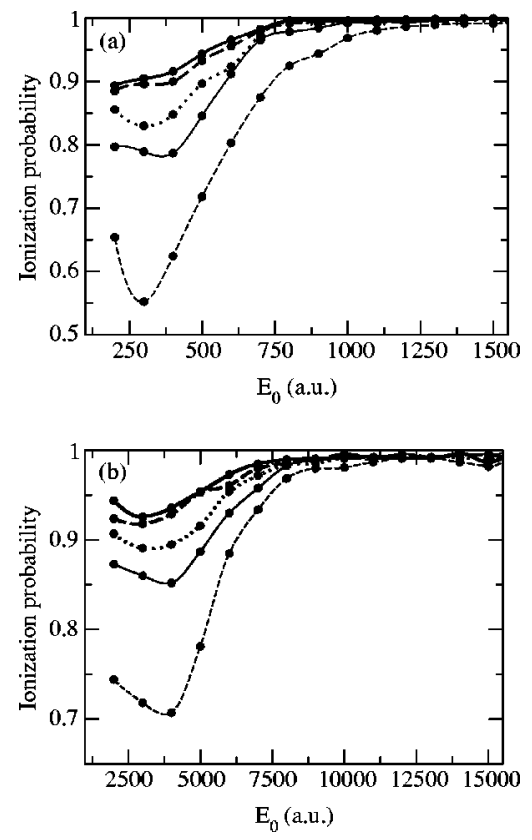

FIG. 6. Ionization probability plotted against the laser electricfield strength for "two-stage" pulses of the form (1-x-4-4) acting on (a) helium $(+)$ and (b) boron $(4+), x$ values examined were 5 (thin dashed line), 50 (thin solid line), 100 (dotted line), 200 (thick dashed line), and 300 cycles (thick solid line). Laser frequencies were again scaled as in Fig. 5(b): (a) $\omega=20.0$ a.u. and (b) $\omega$ $=125.0$ a.u. The probability values were found by averaging over 1000 Monte Carlo trajectories.

same ionization probabilities, and at field strengths scaled by $Z^{3}$ in relation to hydrogen. However, the stabilization dip gets less pronounced with increasing $Z$, and the point at which stabilization ends with respect to $E_{0}$ no longer scales strictly with $Z^{3}$, implying that a more complicated relationship exists between hydrogen and the hydrogenlike ions in the relativistic regime. The reason behind this unexpected change in the stabilization feature itself is not fully understood. The most appealing possibility to us is that, due to the relativistic treatment, there is a limit on the velocity that the electron can attain. Therefore, there is a limit on the speed at which the electron can move away from the nucleus and into the region where it cannot gain much net energy. Thus, due to the relativistic mass shift, the electron-nucleus interaction is longer than given merely by the nonrelativistic scaling law.

Adiabatic-pulse turn-on stages have been found to be an important factor in the onset of stabilization in nonrelativistic treatments. Hence the opportunity is taken to examine the effect that adiabatic field rampings have on ionization probability in our treatment. To this end, the two-stage pulse envelope is employed to aid the system into surviving the "death-valley" region of the turn-on stage, and then to increase the electric-field strength adiabatically for strengths where stabilization can occur. The results of such pulses are shown in Fig. 6 for the cases of helium (+) and boron (4+). The second stage of the field ramping began in each case when the ratio $E_{0} / \omega=5 \%$ of $c$. This was chosen so as to start the adiabatic stage within the stabilization region, having not yet entered the regime where the electron attains 
relativistic velocities. For short field rampings $(x=5)$, the stabilization feature is clearly visible. It turns out to be lower and more pronounced than for the longer pulses, with the point at which $100 \%$ ionization is reached shifted towards higher electric-field strengths. The stabilization feature gets shallower and overall ionization increases with increasing $x$, implying that the dominant factor is the increased time of interaction that is inherent in longer pulses, rather than the adiabatic nature of the ramping. However, adiabaticity does have a strong influence on the dynamics of the system. This is based on the observation that the curves get closer together as $x$ increases, and that for $x=200$ and $x=300$, the curves almost lie on top of one another, despite the 100-cycle difference.

Indeed, we have found that very short field rampings encourage stabilization. Cycles that have field rampings less than between 3-5 cycles in duration tend not to stabilize, whereas larger cycle numbers, while still exhibiting stabilization, tend to have greater ionization probabilities within the stabilization region.

This all leads to two possible scenarios in the almost limiting case of extremely long second-stage field rampings (an infinite second stage would mean that maximum intensity is never reached). One possibility is that the ionization probability in the stabilization regime could carry on increasing with $x$, with decreasing difference between successive curves until it reaches unity in the limiting case (effect of adiabatic ramping is not enough to compensate for length of interaction time). The second, more intriguing possibility is that the ionization probability in the stabilization region never reaches unity. In other words, there may always be a finite possibility of the system surviving, even for almost semiinfinite laser pulses, so the effect of adiabatic ramping is enough to compensate for very long interaction times. Given our classical analysis with turn-on phases up to 300 cycles, we consider it more likely, however, for all practical purposes that the breakdown of stabilization may not be inhibited in the relativistic regime.

\section{CONCLUSIONS}

We began by showing that stabilized single-electron trajectories are increasingly governed by relativistic signatures with rising charge states. This included magnetic recollisions at overall smaller spatial dimensions, which become essentially equal in the laser polarization and propagation directions at larger $Z$. Hydrogenlike ions then turned out to exhibit stabilization in a manner similar to that of hydrogen apart from a scaling law and relativistic signatures. Those similar ionization curves at electrical-field strengths, scaled proportional to $Z^{3}$ from the hydrogen case, can be produced if the laser frequency is scaled by $Z^{2}$. Heavier ions, however, start showing different behavior when extremely high frequencies and intensities are employed because the nonrelativistic scaling law leads to an increase of the achievable electron velocity with $Z$. This involves a substantial reduction of stabilization and was linked to the relativistic constraint on the electron's velocity, i.e., the relativistic mass shift, which gives rise to a limit on the minimum time the electron has to remain in the vicinity of the nucleus. This in turn implies that there is greater energy and angular momentum transfer to the electron at the higher field strengths for the heavier ions, allowing a greater ionization probability.

We have also pointed out that the degree of adiabaticity in the field turn-on stage(s) has a marked effect on the ionization probability within the stabilization region. Stabilization requires pulse turn-on stages of duration 3-5 cycles or longer, and that for very long pulse turn-on above 100 cycles, the variation in the ionization probabilities becomes increasingly small when the cycle numbers grow. Still, however, the eventual breakdown of stabilization could not be avoided because the role of the longer interaction time dominates that of the enhanced adiabaticity.

\section{ACKNOWLEDGMENTS}

L.N.G. acknowledges, with thanks, the financial support of the ERASMUS program of the European Union, and C.H.K. acknowledges the funding by the German Science Foundation (Nachwuchsgruppe within SFB276). C.H.K. has benefited from discussions with J. S. Briggs and H. G. Muller and L.N.G. wishes to thank J. S. Briggs and all members of Theoretische Quantendynamik for their hospitality.
[1] M. Gavrila and J. Z. Kamiński, Phys. Rev. Lett. 52, 613 (1984).

[2] Q. Su, J. H. Eberly, and J. Javanainen, Phys. Rev. Lett. 64, 862 (1990).

[3] V. C. Reed, P. L. Knight, and K. Burnett, Phys. Rev. Lett. 67, 1415 (1991).

[4] V. C. Reed and K. Burnett, Phys. Rev. A 47, R34 (1993).

[5] R. Grobe and C. K. Law, Phys. Rev. A 44, R4114 (1991).

[6] C. K. Law, Q. Su, and J. H. Eberly, Phys. Rev. A 44, 7844 (1991).

[7] M. Protopapas, C. H. Keitel, and P. L. Knight, J. Phys. B 29, L591 (1996).
[8] N. J. Kylstra, A. M. Ermolaev, and C. J. Joachain, J. Phys. B 30, L449 (1997).

[9] R. Taieb, V. Véniard, and A. Maquet, Phys. Rev. Lett. 81, 2882 (1998).

[10] U. W. Rathe, C. H. Keitel, M. Protopapas, and P. L. Knight, J. Phys. B 30, L531 (1997).

[11] N. J. Kylstra, R. A. Worthington, A. Patel, P. L. Knight, J. R. Vázquez de Aldana, and L. Roso, Phys. Rev. Lett. 85, 1835 (2000).

[12] J. R. Vázquez de Aldana, N. J. Kylstra, L. Roso, P. L. Knight, A. Patel, and R. A. Worthington, Phys. Rev. A 64, 013411 (2001). 
[13] A. Patel, M. Protopapas, D. G. Lappas, and P. L. Knight, Phys. Rev. A 58, R2652 (2001).

[14] K. C. Kulander, K. J. Schafer, and J. L. Krause, Phys. Rev. Lett. 66, 2601 (1991).

[15] J. Grochmalicki, M. Lewenstein, and K. Rzążewski, Phys. Rev. Lett. 66, 1038 (1991).

[16] M. Gajda, J. Grochmalicki, M. Lewenstein, and K. Rzążewski, Phys. Rev. A 46, 1638 (1992).

[17] C. H. Keitel and P. L. Knight, Phys. Rev. A 51, 1420 (1995).

[18] M. Gavrila, in Multiphoton Processes, edited by L. F. DiMauro, R. R. Freemen, and K. C. Kulander, AIP Conf. Proc. No. 525 (AIP, Melville, NY, 2000), p. 103.

[19] M. Pont and M. Gavrila, Phys. Rev. Lett. 65, 2362 (1990).

[20] H. R. Reiss, Opt. Express 8, 99 (2000).

[21] C. F. D. Faria, A. Fring, and R. Schrader, J. Phys. B 33, 1675 (2000).

[22] N. J. van Druten, R. C. Constantinescu, J. M. Schins, H. Nieuwenhuize, and H. G. Muller, Phys. Rev. A 55, 622 (1997).

[23] D. Bauer and F. Ceccherini, Phys. Rev. A 60, 2301 (1999).

[24] F. Ceccherini, D. Bauer, and P. Mulser, Laser Part. Beams 18, 449 (2000).

[25] A. Talebpour, K. Vijayalakshmi, A. D. Bandrauk, T. T. Nguyen-Dang, and S. L. Chin, Phys. Rev. A 62, 042708
(2000).

[26] M. Protopapas, C. H. Keitel, and P. L. Knight, Rep. Prog. Phys. 60, 389 (1997).

[27] S. X. Hu and C. H. Keitel, Phys. Rev. A 63, 053402 (2001).

[28] D. B. Milošević, S. X. Hu, and W. Becker, Phys. Rev. A 63, R011403 (2001).

[29] N. J. Kylstra, R. M. Potvliege, and C. J. Joachain, J. Phys. B 34, L55 (2001).

[30] K. Z. Hatsagortsyan and C. H. Keitel, Phys. Rev. Lett. 86, 2277 (2001).

[31] J. Bestle, V. M. Akulin, and W. P. Schleich, Phys. Rev. A 48, 746 (1993).

[32] R. Abrines and I. C. Percival, Proc. Phys. Soc. London 88, 861 (1966).

[33] H. Goldstein, Classical Mechanics, 2nd ed. (Addison-Wesley, Reading, MA, 1980).

[34] J. H. Eberly, Phys. Rev. A 42, 5750 (1990).

[35] R. E. Olson and A. Salop, Phys. Rev. A 16, 531 (1977).

[36] J. S. Cohen, Phys. Rev. A 26, 3008 (1982).

[37] G. Bandarage and R. Parson, Phys. Rev. A 41, 5878 (1990).

[38] D. J. Urbach and C. H. Keitel, Phys. Rev. A 61, 043409 (2000). 